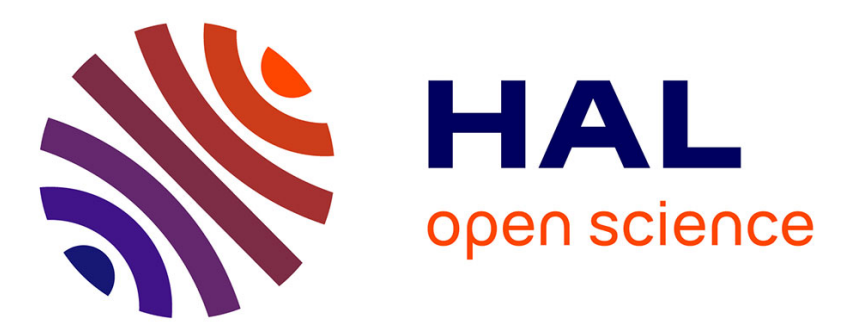

\title{
An Auction Framework for Spectrum Allocation with Interference Constraint in Cognitive Radio Networks
} Lin Chen, Stefano Iellamo, Marceau Coupechoux, Philippe Godlewski

\section{To cite this version:}

Lin Chen, Stefano Iellamo, Marceau Coupechoux, Philippe Godlewski. An Auction Framework for Spectrum Allocation with Interference Constraint in Cognitive Radio Networks. IEEE INFOCOM, Mar 2010, San Diego, États-Unis. pp.1-9. hal-01144503

\section{HAL Id: hal-01144503 https://hal-imt.archives-ouvertes.fr/hal-01144503}

Submitted on 21 Apr 2015

HAL is a multi-disciplinary open access archive for the deposit and dissemination of scientific research documents, whether they are published or not. The documents may come from teaching and research institutions in France or abroad, or from public or private research centers.
L'archive ouverte pluridisciplinaire HAL, est destinée au dépôt et à la diffusion de documents scientifiques de niveau recherche, publiés ou non, émanant des établissements d'enseignement et de recherche français ou étrangers, des laboratoires publics ou privés. 


\section{An Auction Framework for Spectrum Allocation with Interference Constraint in Cognitive Radio Networks}

\author{
Lin Chen* \\ Stefano Iellamo ${ }^{\dagger}$ \\ *Department of Computer Science and Networking \\ Telecom ParisTech - LTCI CNRS 5141 \\ 46 Rue Barrault, Paris 75013, France \\ \{lchen, coupecho, godlewsk\}@enst.fr
}

\author{
Marceau Coupechoux* \\ Philippe Godlewski* \\ ${ }^{\dagger}$ Dipartimento di Elettronica e Informazione \\ Politecnico di Milano \\ Piazza L. da Vinci 32, Milan, Italy \\ dajelvento@gmail.com
}

\begin{abstract}
Extensive research in recent years has shown the benefits of cognitive radio technologies to improve the flexibility and efficiency of spectrum utilization. This new communication paradigm, however, requires a well-designed spectrum allocation mechanism. In this paper, we propose an auction framework for cognitive radio networks to allow unlicensed secondary users (SUs) to share the available spectrum of licensed primary users (PUs) fairly and efficiently, subject to the interference temperature constraint at each PU. To study the competition among SUs, we formulate a non-cooperative multiple-PU multiple-SU auction game and study the structure of the resulting equilibrium by solving a non-continuous two-dimensional optimization problem. A distributed algorithm is developped in which each SU updates its strategy based on local information to converge to the equilibrium. We then extend the proposed auction framework to the more challenging scenario with free spectrum bands. We develop an algorithm based on the no-regret learning to reach a correlated equilibrium of the auction game. The proposed algorithm, which can be implemented distributedly based on local observation, is especially suited in decentralized adaptive learning environments as cognitive radio networks. Finally, through numerical experiments, we demonstrate the effectiveness of the proposed auction framework in achieving high efficiency and fairness in spectrum allocation.
\end{abstract}

\section{INTRODUCTION}

Cognitive radio [1] has emerged in recent years as a promising paradigm to enable more efficient and spectrum utilization. Apart from the conventional command and control model, three more flexible spectrum management models are presented in [2], namely, exclusive use (or operator sharing), commons and shared use of primary licensed spectrum. In the last model, unlicensed secondary users (SUs) are allowed to access the spectrum of licensed primary users (PUs) in an opportunistic way. In such a model, a well-designed spectrum allocation mechanism is crucial to achieve efficient spectrum usage and harmonious coexistence of PUs and SUs. On one hand, the radio resource allocation mechanism should ensure that the spectrum resource (unused by PUs) is allocated efficiently and fairly among SUs. On the other hand, the communication of PUs should not be disturbed by the SUs.

In this paper, we tackle the challenging research problem of designing efficient spectrum allocation mechanism for cognitive radio networks. We consider a generic network scenario in which multiple PUs and SUs coexist. To use the spectrum resource efficiently, the SUs share the available spectrum of

\footnotetext{
* This work is supported by the project TEROPP (technologies for TERminal in OPPortunistic radio applications) funded by the French National Research Agency (ANR).
}

the PUs under the condition that the interference temperature constraint [3] is always satisfied at each PU, i.e. the total received power of the SUs at each PU should be kept under some threshold in order to protect the PU's traffic. The considered scenario can represent various network scenarios, e.g. the PUs are the access points of a mesh network and the SUs are the mobile devices.

In our work, we develop an auction framework to allow SUs to share the available spectrum of PUs. Under the proposed auction framework, each PU acts as a resource provider by (1) announcing a price and a reserve bid (2) allocating the received power as a function of the bids submitted by SUs. Each SU acts as a customer by (1) submitting a two-dimensional bid indicating which PU to bid for resource and how much to bid (2) paying the chosen PU an amount of payment proportional to the allocated resource and the announced price. To study the competition among SUs, we formulate a noncooperative auction game and study the structure of the resulting Nash equilibrium (NE) by solving a non-continuous twodimensional optimization problem. A distributed algorithm is developped in which each SU updates its strategy based on local information to converge to the NE. Our analysis can serve as a decision and control framework for the SUs to exploit the underutilized spectrum resource.

We then extend the proposed auction framework to the more challenging scenario with free spectrum bands. In this context, a SU should strike a balance between accessing a free spectrum band with more interference if the competitors take the same strategy, and paying more for communication gains by staying with a licensed band. We show that the ping-pong effect may occur under the best-response update, i.e., a SU keeps switching between the free band and a licensed band. To eliminate the ping-pong effect, we develop an algorithm based on the no-regret learning [4] to reach a correlated equilibrium (CE) [5] of the auction game. The proposed algorithm, which can be implemented distributedly and requires only local observation, is especially suited in decentralized adaptive learning environments as cognitive radio networks.

Due to their perceived fairness and allocation efficiency [6], auctions are among the best-known market-based mechanisms to allocate spectrum [7], [8], [9], [10], [11], [12]. In most proposed auctions, the spectrum resource is treated as goods in traditional auctions studied by economists, i.e., one licensed band (or a collection of multiple bands) is awarded to one SU. However, spectrum auction differs from conventional 
auctions in that it has to address radio interference. Spectrum auction is essentially a problem of interference-constrained resource allocation. Only a few papers have discussed spectrum auctions under interference constraint, among which [11] and [12] studied conflict-free spectrum allocation with high spectrum efficiency. [10] developed an auction-based spectrum sharing framework to allow a single spectrum manager to share its spectrum with a group of users, subject to the interference temperature constraint at the measurement point, a requirement proposed by FCC in [3]. Based on the same model as [10], our work is among the relative few that investigate the interference-constraint radio resource allocation problem under the auction framework. Compared with previous work, we make the following key contributions:

- Existing auction mechanisms mainly focus on singlePU scenario with very limited analytical and numerical studies on multiple-PU case. Our work, however, conduct an in-depth analysis on the spectrum auction for multiple PUs to allocate their spectrum to multiple SUs efficiently and fairly. As a distinctive feature of the proposed auction framework, the SUs' strategy (bid) is two-dimensional and non-continuous, leading to a competition scenario with more complex interactions among players and requiring an original study of the resulting equilibrium.

- We investigate the spectrum auction with free spectrum bands and develop a distributed adaptive algorithm based on no-regret learning to converge to a $\mathrm{CE}$ of the auction game. To the best of our knowledge, our work is the first to adapt the auction framework to address the spectrum sharing problem in heterogeneous environments with both licensed and free bands.

The rest of this paper is structured as follows. Section II presents our system model and auction framework followed by the formulation of the non-cooperative auction game. Section III solves the auction game and analyzes the structural properties of the resulting NE. Section IV extends our auction framework to the more challenging scenario with free spectrum bands. Simulation results are presented in Section V. Section VI concludes the paper.

\section{System Model AND Spectrum Auctions}

This section introduces the notation and the system model of our work, followed by the presentation of the proposed spectrum auction framework and the formulation of the auction game under the framework.

\section{A. Cognitive radio network model}

We consider a cognitive radio network consisting of a set of primary users referred to as PUs and a set of secondary transmitter-receiver pairs referred to as secondary users or SUs. We use $\mathcal{N}=\{1,2, \cdots, N\}$ and $\mathcal{M}=\{1,2, \cdots, M\}$ to denote the PU set and the SU set, respectively. We use $S_{i}$ and $D_{i}$ to denote the transmitter and the receiver of SU $i \in \mathcal{M}$. Each PU $n \in \mathcal{N}$ operates on a spectrum band $n$ with bandwidth $B_{n}$ that is non-overlapped with the spectrum bands of other PUs, i.e. $n_{1} \bigcap n_{2}=\Phi, \forall n_{1}, n_{2} \in \mathcal{N}$. ${ }^{1}$

\footnotetext{
${ }^{1}$ The extension of our analysis to the more competitive scenario where the PUs' bands are overlapped with each other is left for future work.
}

SU $i$ 's valuation of the spectrum is defined by a utility function $U_{i}\left(\gamma_{i}\right)$, where $\gamma_{i}$ is the received signal-to-interferenceplus-noise ratio (SINR) at SU i's receiver $D_{i} . U_{i}\left(\gamma_{i}\right)$ characterizes the application payoff (e.g. satisfaction level) of SU $i$ from SINR $\gamma_{i}$. We assume $U_{i}\left(\gamma_{i}\right)$ is continuously differentiable, strictly increasing and concave in $\gamma_{i}$ with $U_{i}(0)=0$. For each SU $i$, the received SINR using PU $n$ 's band is given by

$$
\gamma_{i}=\frac{p_{i} h_{i i}}{n_{0} B_{n}+\sum_{j \neq i} p_{j} h_{j i}},
$$

where $p_{i}$ denotes SU $i$ 's transmission power, $h_{j i}$ denotes the channel gain from SU $j$ 's transmitter $S_{j}$ to $\mathrm{SU} i$ 's receiver $D_{i}, n_{0}$ denotes the background noise power spectral density.

In the considered scenario, to ensure that the transmissions of PUs are not significantly degraded by the SUs, an interference temperature constraint is imposed such that the total received power of SUs at PU $n$ must satisfy

$$
\sum_{i=1}^{M} p_{i} g_{i n} \leq P_{n} \quad \forall n \in \mathcal{N},
$$

where $g_{i n}$ is the channel gain from $S_{i}$ to $\mathrm{PU} n, P_{n}$ is the tolerable interference threshold at PU $n$.

\section{B. Spectrum auction framework}

We apply auction mechanisms to tackle the spectrum allocation problem. By definition, an auction is a decentralized market mechanism for allocating resources and can be formulated as a non-cooperative game, where players are bidders, strategies are bids, both allocations and payments are functions of bids. A well-known auction is the Vickrey-Clarke-Groves (VCG) auction [6], which is shown to have social optimal outcome. However, the VCG auction requires global information to perform centralized computations. To overcome this limitation, two one-dimensional share auction mechanisms, namely the SINR auction and the power auction are proposed in [10] to study the spectrum allocation problem in single-PU networks. In the following, we extend the work of [10] to the multiple-PU scenario by proposing the two-dimensional SINR and power auction, as shown in Algorithm $1 .^{2}$

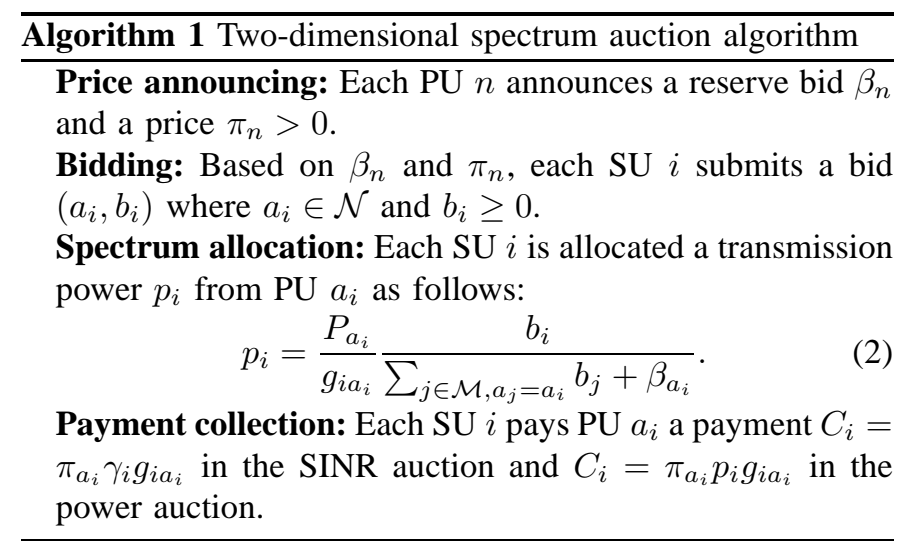

\footnotetext{
${ }^{2}$ In our study, we assume that SUs are honest, and indeed make the payments. We do not consider the issue of payment enforcement, which may require a separate mechanism and is beyond the scope of the paper.
} 
Under the above auction framework, the received SINR of $\mathrm{SU} i$ is

$$
\gamma_{i}=\frac{P_{a_{i}} \frac{h_{i i}}{g_{i a_{i}}} b_{i}}{n_{0} B_{a_{i}}\left(\sum_{j \in \mathcal{M}, a_{j}=a_{i}} b_{j}+\beta_{a_{i}}\right)+\sum_{j \in \mathcal{M}, a_{j}=a_{i}, j \neq i} P_{a_{i}} \frac{h_{j i}}{g_{j a_{i}}} b_{j}} .
$$

In contrast to [10] where SUs are charged the same price per unit SINR, we apply the economic concept of price discrimination in the proposed SINR auction by imposing $g_{i a_{i}}$ as a user-dependent pricing factor on $\mathrm{SU} i$. The design rationale is that for two SUs choosing the same PU, the SU causing more interference at the PU should be charged more per unit SINR than the SU causing less interference. As we will show via numerical experiments, this feature is especially suited in multi-PU case by resulting a more balanced equilibrium. For the power auction, noticing that the received power of SU $i$ at PU $a_{i}$ is $p_{i} g_{i a_{i}}$, the auction scheme actually inplements a pricing policy under which a price $\pi_{n}$ per unit received power is imposed by PU $n$ to the SUs connecting to it.

\section{Non-cooperative spectrum auction game formulation}

Under the proposed auction framework, we model the interaction among SUs as a non-cooperative spectrum auction game, denoted as $G_{N S A}$ and $G_{N P A}$ for the SINR and power auction, respectively. ${ }^{3}$ Let $s_{i}=\left(a_{i}, b_{i}\right)$ denote the strategy of SU $i$ and $s_{-i}$ denote the strategy of the SUs except $i$, given the price vector $\pi=\left(\pi_{n}, n \in \mathcal{N}\right)$, each SU $i$ chooses its strategy $s_{i}$ to maximize his surplus function defined as follows:

$$
S_{i}\left(s_{i}, s_{-i}\right)=U_{i}\left(\gamma_{i}\left(s_{i}, s_{-i}\right)\right)-C_{i}\left(s_{i}, s_{-i}\right) .
$$

The resulting non-cooperative SINR (power) auction game can then be defined formally as:

$$
G_{N S A}\left(G_{N P A}\right): \max _{s_{i}=\left(a_{i}, b_{i}\right), a_{i} \in \mathcal{N}, b_{i} \geq 0} S_{i}\left(s_{i}, s_{-i}\right), \forall i \in \mathcal{M} .
$$

The solution of the auction game is characterized by a Nash Equilibrium (NE), a strategy profile $\mathbf{s}^{*}=\left(s_{i}^{*}, s_{-i}^{*}\right)$ from which no player has incentive to deviate unilaterally [13], i.e.,

$$
S_{i}\left(s_{i}^{*}, s_{-i}^{*}\right) \geq S_{i}\left(s_{i}, s_{-i}^{*}\right), \quad \forall i \in \mathcal{M}, \forall a_{i} \in \mathcal{N}, \forall b_{i} \geq 0 .
$$

As a distinguished feature from the single-PU auction, the auction framework proposed in our work is two-dimensional and involves both PU selection and bid adjustment, which leads to a competition scenario with more complex interactions among players. Consequently, characterizing structural properties of the auction game in our context requires an original study of the game equilibria that cannot draw on existing wellknown results, as will be shown in later analysis.

\section{Solving the Auction Game: NE Analysis}

In this section, we solve the auction game by deriving the $\mathrm{NE}$ of the game and study the structure properties of the NE. To this end, we focus on the following optimization problem

\footnotetext{
${ }^{3}$ In this work, we do not consider the PUs as players. A significant extension of our work presented in this paper is to model the spectrum auction as a Stackelberg game, in which the PUs are the leaders that choose their strategy (price) first, and the SUs are the followers that respond by choosing their strategies (bids) accordingly, knowing the leaders' strategies [13]. We leave this extension of exploring the Stackelberg game for future work.
}

faced by each $\mathrm{SU} i$ in the spectrum auction game, given the price of PUs $\pi=\left\{\pi_{n}, n \in \mathcal{N}\right\}$ and strategies of others $s_{-i}$ :

$$
s_{i}^{*}=\left(a_{i}^{*}, b_{i}^{*}\right)=\underset{s_{i}}{\operatorname{argmax}} S_{i}\left(s_{i}, s_{-i}\right),
$$

which, according to the following lemma, can be written as

$$
s_{i}^{*}=\left(a_{i}^{*}, b_{i}^{*}\right)=\underset{a_{i} \in \mathcal{N}}{\operatorname{argmax}} \underset{b_{i} \geq 0}{\operatorname{argmax}} S_{i}\left(s_{i}, s_{-i}\right) .
$$

Lemma 1. $\max _{\left(a_{i}, b_{i}\right)} S_{i}\left(s_{i}, s_{-i}\right)=\max _{a_{i} \in \mathcal{N}} \max _{b_{i} \geq 0} S_{i}\left(s_{i}, s_{-i}\right)$.

Proof: On one hand, it follows from (4) that

$$
S_{i}\left(\left(a_{i}^{*}, b_{i}^{*}\right), s_{-i}\right) \geq \max _{a_{i} \in \mathcal{N}} \max _{b_{i} \geq 0} S_{i}\left(\left(a_{i}, b_{i}\right), s_{-i}\right) .^{4}
$$

On the other hand, we have

$$
\begin{aligned}
\max _{a_{i} \in \mathcal{N}} \max _{b_{i} \geq 0} S_{i}\left(\left(a_{i}, b_{i}\right), s_{-i}\right) & \geq \max _{b_{i} \geq 0} S_{i}\left(\left(a_{i}^{*}, b_{i}\right), s_{-i}\right) \\
& \geq S_{i}\left(\left(a_{i}^{*}, b_{i}^{*}\right), s_{-i}\right) .
\end{aligned}
$$

Combining the above results completes our proof.

\section{A. SINR auction}

We start with the SINR auction game. Unlike the singlePU auction studied in [10], where each SU maximizes its surplus function over its bid only, the SU optimization problem in the multiple-PU case is a joint two-dimensional problem over the submitted bid and the PU to whom the SU bids for spectrum. To solve the SUs' optimization problem, a straightforward way to find $\left(a_{i}^{*}, b_{i}^{*}\right)$ is to search over all possible PU settings and perform optimization over bid for every setting, which is computationally intensive and makes the resulting $\mathrm{NE}$ intractable. In our analysis, we overcome this technical difficulty by decomposing the two-dimensional optimization problem based on the structural properties of the surplus function, detailed in Lemma 2.

Lemma 2. For each $S U$, given $\pi$ and $s_{-i}$, it holds that

$$
a_{i}^{*}=\underset{n \in \mathcal{N}}{\operatorname{argmax}} S_{i}\left(\gamma_{i n}^{*}\right)=\underset{n \in \mathcal{N}}{\operatorname{argmax}} U_{i}\left(\gamma_{i n}^{*}\right)-\pi_{n} g_{i n} \gamma_{i n}^{*},
$$
where $\gamma_{i n}^{*}=\min \left\{U_{i}^{\prime-1}\left(\pi_{n} g_{i n}\right), P_{n} h_{i i} /\left(n_{0} B_{n} g_{i n}\right)\right\}, \forall n \in \mathcal{N}$.

Proof: Let $\gamma_{i n}$ denote the SINR of SU $i$ when connecting to PU $n$, recall (3), we can show that:

1) $\gamma_{i n}$ is upper-bounded by $P_{n} h_{i i} /\left(n_{0} B_{n} g_{i n}\right)$;

2) For $\gamma_{i n} \leq P_{n} h_{i i} /\left(n_{0} B_{n} g_{i n}\right)$, there is an one-to-one mapping between $\gamma_{i n}$ and $b_{i}$.

From Lemma 1, the optimization problem of $\mathrm{SU} i$ is thus equivalent to the following one:

$$
\max _{n \in \mathcal{N}} \max _{\gamma_{i n} \leq \frac{P_{n} h_{i i}}{n_{0} B_{n} g_{i n}}} S_{i}\left(n, \gamma_{i n}\right) \text {. }
$$

Moreover, when choosing PU $n, S_{i}$ can be written as a function of $\gamma_{i n}$ as

$$
S_{i}\left(\gamma_{i n}\right)=U_{i}\left(\gamma_{i n}\right)-\pi_{n} g_{i n} \gamma_{i n},
$$

whose derivative is

$$
\frac{\partial S_{i}}{\partial \gamma_{i n}}=U_{i}^{\prime}\left(\gamma_{i n}\right)-\pi_{n} g_{i n}
$$

Following the concavity of $U_{i}, U_{i}^{\prime}$ is monotonously decreasing in $\gamma_{i n}$. Hence $S_{i}$ is a quasi-concave function of $\gamma_{i n}$, thus has a unique global maximizer $\gamma_{i n}^{*}=$

${ }^{4}$ For the sake of simplicity, in case of non-ambiguity, we note $S_{i}\left(\left(a_{i}^{*}, b_{i}^{*}\right), s_{-i}\right)$ as a function of $s_{i}$, i.e. $S_{i}\left(s_{i}\right)$ or $S_{i}\left(a_{i}^{*}, b_{i}^{*}\right)$. 
$\min \left\{U_{i}^{\prime-1}\left(\pi_{n} g_{i n}\right), P_{n} h_{i i} /\left(n_{0} B_{n} g_{i n}\right)\right\}$. The maximum of $S_{i}$ under PU $n$ is given by $S_{i}\left(\gamma_{i n}^{*}\right)$. It then follows that

$$
a_{i}^{*}=\underset{n \in \mathcal{N}}{\operatorname{argmax}} S_{i}\left(\gamma_{i n}^{*}\right)=\underset{n \in \mathcal{N}}{\operatorname{argmax}} U_{i}\left(\gamma_{i n}^{*}\right)-\pi_{n} g_{i n} \gamma_{i n}^{*},
$$

where $\gamma_{i n}^{*}=\min \left\{U_{i}^{\prime-1}\left(\pi_{n} g_{i n}\right), P_{n} h_{i i} /\left(n_{0} B_{n} g_{i n}\right)\right\}$.

Specifically, when $\pi_{n}$ is significantly large, more precisely, $\pi_{n} g_{\text {in }} \geq U_{i}^{\prime}\left(P_{n} h_{i i} / n_{0} B_{n} g_{i n}\right), \forall n \in \mathcal{N}, \forall i \in \mathcal{M}$, Lemma 2 can be simplified to Corollary 1 .

Corollary 1. If $\pi_{n} g_{\text {in }} \geq U_{i}^{\prime}\left(P_{n} h_{i i} / n_{0} B_{n} g_{i n}\right), \forall n \in \mathcal{N}, \forall i \in$ $\mathcal{M}$, it holds that $a_{i}^{*}=\operatorname{argmin}_{n \in \mathcal{N}} \pi_{n} g_{i n}$.

Proof: Recall that $U_{i}\left(\gamma_{i}\right)$ is concave in $\gamma_{i}$, $\pi_{n} g_{i n} \geq U_{i}^{\prime}\left(P_{n} h_{i i} / n_{0} B_{n} g_{i n}\right)$ leads to $U_{i}^{\prime-1}\left(\pi_{n} g_{i n}\right) \leq$ $P_{n} h_{i i} /\left(n_{0} B_{n} g_{i n}\right)$. It then follows from Lemma 2 that $\gamma_{i n}^{*}=U_{i}^{\prime-1}\left(\pi_{n} g_{i n}\right)$ and

$$
\begin{aligned}
a_{i}^{*} & =\underset{n \in \mathcal{N}}{\operatorname{argmax}} S_{i}\left(U_{i}^{\prime-1}\left(\pi_{n} g_{i n}\right)\right) \\
& =\underset{n \in \mathcal{N}}{\operatorname{argmax}} U_{i}\left(U_{i}^{\prime-1}\left(\pi_{n} g_{i n}\right)\right)-\pi_{n} g_{i n} U_{i}^{\prime-1}\left(\pi_{n} g_{i n}\right) .
\end{aligned}
$$

Let $x=\pi_{n} g_{i n}$, regard $S_{i}=U_{i}\left(U_{i}^{\prime-1}(x)\right)-x U_{i}^{\prime-1}(x)$ as a function of $x$, after some mathematical operations, we have

$$
\frac{\partial S_{i}}{\partial x}=-U_{i}^{\prime-1}(x),
$$

which, following the concavity of $U_{i}$, is non-positive. $S_{i}(x)$ is thus non-increasing in $x$. Hence

$$
a_{i}^{*}=\underset{n \in \mathcal{N}}{\operatorname{argmax}} S_{i}\left(U_{i}^{\prime-1}\left(\pi_{n} g_{i n}\right)\right)=\underset{n \in \mathcal{N}}{\operatorname{argmin}} \pi_{n} g_{i n},
$$

which concludes our proof.

If we denote $\pi_{n} g_{i n}$ as the effective price for SU $i$ when choosing PU $n$, Corollary 1 states that $\mathrm{SU} i$ always chooses the PU with the minimum effective price.

As the key results of this subsection, we have demonstrated that in the SINR auction game, the choice of PU only depends on the effective price set by PUs. Consequently, the optimization problem of each $\mathrm{SU} i$ can be decomposed into two sub-problems, which can be performed sequentially:

1) $i$ chooses PU $a_{i}^{*}$ based on the effective price of PUs and stay with PU $a_{i}^{*}$;

2) $i$ performs bid optimization by adjusting its bid submitted to PU $a_{i}^{*}$, which is degenerated into single-PU case.

The following theorem on the NE of the SINR auction game is then immediate whose proof follows straightforwardly from that of Theorem 1 and Proposition 6 in [10].

Theorem 1. For the SINR auction with $\beta_{n}>0, \forall n \in \mathcal{N}$, there exists a threshold price vector $\pi_{t h}^{s}=\left\{\pi_{t h, n}^{s}, n \in \mathcal{N}\right\}$ such that if the price vector $\pi>\pi_{t h}^{s},{ }^{5}$ a NE exists to which the best response update converges. The NE is unique if $a_{i}^{*}$ $i$ singleton for every $S U i$. On the other hand, if there exists some $n_{0} \in \mathcal{N}$ such that $\pi_{n_{0}} \leq \pi_{t h, n_{0}}^{s}$, there is no $N E$.

\section{B. Power auction}

In this subsection, we turn to the power auction game. As the payment function $C_{i}$ in the power auction has a different structure to that in the SINR auction (i.e. $C_{i}$ is a function of $p_{i}$ instead of $\gamma_{i}$ ), the decomposition in the previous analysis on the SINR auction is no more applicable here. To characterize

\footnotetext{
${ }^{5}$ Throughout the paper, the inequality between two vectors is defined as the inequality in all components of the vectors.
}

the equilibrium of the power auction game, we make the following approximation in the subsequent analysis:

$$
\sum_{a_{j}=a_{i}, j \neq i} b_{j} \gg b_{i}, \forall i \in \mathcal{M} \text {, or equivalently, } \sum_{s_{j}=a_{i}, j \neq i} b_{j} \sim \sum_{s_{j}=a_{i}} b_{j} .
$$

The approximation (5) is accurate in large systems where the bid variation of any individual player has neglectable influence on the system state. More specifically, under (5), the impact of $b_{i}$ on the interference at the receiver $D_{i}$, denoted as $I_{i}$, can be neglected, in other words, $I_{i}$ can be regarded independent w.r.t. $b_{i}$. The utility function of SU $i$ can then be written as:

$$
S_{i}=U_{i}\left(\gamma_{i}\right)-\frac{\pi_{a_{i}} g_{i a_{i}} I_{i}}{h_{i i}} \gamma_{i} .
$$

where $I_{i}=n_{0} B_{a_{i}}+\sum_{j \in \mathcal{M}, j \neq i, a_{j}=a_{i}} p_{j} h_{j i}$.

To solve the power auction game, we transform the original game $G_{N P A}$ into another game $G_{N P A}^{\prime}$ in which the strategy of SU $i$ is $\left(a_{i}, \gamma_{i}\right)$ instead of $\left(a_{i}, b_{i}\right)$ in $G_{N P A}$. Under the approximation (5), $\gamma_{i}$ can be regarded as a linear function of $b_{i}$. As $I_{i}$ is independent w.r.t. $b_{i}$, any unilateral change in $b_{i}$ can be transformed into related change in $\gamma_{i}$ without any influence on $\gamma_{-i}$. Thus the original game $G_{N P A}$ is equivalent to the transformed game $G_{N P A}^{\prime}$, formally expressed as

$$
G_{N P A}^{\prime}: \quad \max _{s_{i}=\left(a_{i}, \gamma_{i}\right)} S_{i}\left(s_{i}, s_{-i}\right), i \in \mathcal{M} .
$$

We now concentrate on the new game $G_{N P A}^{\prime}$. Performing the same analysis as Lemma 1 and Corollary 1 by noticing that $I_{i} \geq n_{0} B_{a_{i}}$, we have the following result that decouples the PU selection and the adjustment of $\gamma_{i}$ in $G_{N P A}^{\prime}$.

Lemma 3. If $\pi_{n} g_{i n} n_{0} B_{n} / h_{i i} \geq U_{i}^{\prime}\left(P_{n} h_{i i} / n_{0} g_{i n}\right), \forall n \in$ $\mathcal{N}, \forall i \in \mathcal{M}$, it holds that $a_{i}^{*}=\operatorname{argmin}_{n \in \mathcal{N}} \pi_{n} g_{i n} I_{i} / h_{i i}$.

Compared with the SINR auction game where the effective price imposed by PU $n$ to $\mathrm{SU} i$ is $\pi_{n} g_{i n}$, in the power auction game, the corresponding effective price becomes $\pi_{n} g_{i n} I_{i} / h_{i i}$. Lemma 3 states that SU $i$ always chooses the PU with the minimum effective price. Armed with Lemma 3, we can then establish the existence of $\mathrm{NE}$ in $G_{N P A}^{\prime}$ under the condition that the prices set by PUs are sufficiently high.

Theorem 2. Under the approximation (5) and the condition in Lemma 3, $G_{N P A}^{\prime}$ admits a NE.

Proof: For any $\mathrm{SU} i$, under the strategy of others $s_{-i}=$ $\left(a_{-i}, \gamma_{-i}\right)$, it follows from Lemma 3 that $i$ chooses PU $a_{i}^{*}=$ $\min _{n \in \mathcal{N}} \pi_{n} g_{i n} I_{i} / h_{i i}$, i.e., for any $a_{i}^{\prime} \neq a_{i}^{*}$, it holds that

$$
\frac{\pi_{a_{i}^{*}} h_{i a_{i}^{*}} I_{i}\left(a_{i}^{*}\right)}{h_{i i}} \leq \frac{\pi_{a_{i}^{\prime}} h_{i a_{i}^{\prime}} I_{i}\left(a_{i}^{\prime}\right)}{h_{i i}} .
$$

It then follows that for any $\gamma_{i} \geq 0$

$$
\begin{aligned}
S_{i}\left(a_{i}^{*}, \gamma_{i}\right)=U_{i}\left(\gamma_{i}\right)-\frac{\pi_{a_{i}^{*}} h_{i a_{i}^{*}} I_{i}\left(a_{i}^{*}\right)}{h_{i i}} \gamma_{i} \geq \\
U_{i}\left(\gamma_{i}\right)-\frac{\pi_{a_{i}^{\prime}} h_{i a_{i}^{\prime}} I_{i}\left(a_{i}^{\prime}\right)}{h_{i i}} \gamma_{i}=S_{i}\left(a_{i}^{\prime}, \gamma_{i}\right),
\end{aligned}
$$

which implies that given the opponents' strategy, choosing PU $a_{i}^{*}$ is always the dominating strategy for any $\gamma_{i}$.

On the other hand, performing the same analysis as Lemma 1, we can show that in $G_{N P A}^{\prime}$,

$$
\max _{\left(a_{i}, \gamma_{i}\right)} S_{i}\left(s_{i}, s_{-i}\right)=\max _{\gamma_{i}} \max _{a_{i}} S_{i}\left(s_{i}, s_{-i}\right) .
$$

The optimization problem for SU $i$ thus becomes

$$
\max _{\left(a_{i}, \gamma_{i}\right)} S_{i}\left(s_{i}, s_{-i}\right)=\max _{\gamma_{i}} S_{i}\left(a_{i}^{*}, \gamma_{i}\right) \text {, }
$$


in which the utility function of SU $i$ is $S_{i}\left(a_{i}^{*}, \gamma_{i}\right)$, which is concave in $\gamma_{i}$. Furthermore, it follows from $I_{i} \geq n_{0} B_{n}$ and $p_{i} \leq P_{n} / g_{i a_{i}}$ when SU $i$ chooses PU $n$ that $\gamma_{i} \leq$ $\max _{n \in \mathcal{N}} h_{i i} P_{n} /\left(g_{i a_{i}} n_{0} B_{n}\right)$. Thus the strategy space $\gamma=$ $\left(\gamma_{i}, i \in \mathcal{M}\right)$ is a nonempty, convex, and compact set. It then follows from Theorem 1 in [14] that $G_{N P A}^{\prime}$ admits a NE.

Due to the complexity of the power auction game in which each SU has to solve a two-dimentional, non-continuous and non-decomposable optimization problem, we do not have a formal proof of the uniqueness of the $\mathrm{NE}$ and the convergence under the best response update. However, our experiment results show that the convergence is achieved in the vast majority of cases (cf. Section V-C).

\section{The two-level game model}

To get more insight on the structure of the auction game, we introduce and analyze in this subsection the following twolevel game model: the lower level bidding game under fixed PU setting (Definition 1) and the higher level PU selection game (Definition 2).

Definition 1. Given a fixed $P U$ setting $\mathbf{a}=\left\{a_{i}, i \in \mathcal{M}\right\}$, the bidding game, denoted as $G_{N S A}^{B}(\mathbf{a})$ and $G_{N P A}^{B}(\mathbf{a})$ for the SINR and power auction respectively, is a tuple $\left(\mathcal{M}, \mathcal{A},\left\{S_{i}, i \in \mathcal{M}\right\}\right)$, where the $S U$ set $\mathcal{M}$ is the player set, $\mathcal{A}=[0,+\infty)^{M}$ is the strategy set, $\left\{S_{i}\right\}$ is the utility function set with $S_{i}$ being the surplus function. Each player $(S U) i$ selects its strategy (bid) $b_{i} \geq 0$ to maximize its utility $S_{i}$.

The above defined bidding game can be analyzed in the same way as the single-PU bidding game presented in [10] with the following result on the NE.

Lemma 4. For the SINR auction (for the power auction under the approximation (5)) with $\beta_{n}>0, \forall n \in \mathcal{N}$, there exists a threshold price vector $\pi_{t h}^{s b}(\mathbf{a})\left(\pi_{t h}^{p b}(\mathbf{a})\right)$ such that there exists a NE to which the best response update converges if the price vector $\pi>\pi_{t h}^{s b}(\mathbf{a})\left(\pi>\pi_{t h}^{p b}(\mathbf{a})\right)$, there is no NE otherwise.

Proof: The proof for the SINR auction follows immediately from Theorem 1 and Proposition 6 in [10]. For the power auction, we show that under the condition in the lemma, the best response function has the same structure as that in the SINR auction in [10] whose convergence to NE is proven (Theorem 1 in [10]). To this end, recall that under (5), the utility function can be written as

$$
S_{i}=U_{i}\left(\gamma_{i}\right)-\frac{\pi g_{i 0} I_{i}}{h_{i i}} \gamma_{i}
$$

where $I_{i}$ is independent of $b_{i}$. For each SU $i$, we can solve the best response $b_{i}=B\left(b_{-i}\right)$ as follows:

$$
\begin{cases}b_{i}=+\infty & \text { if } \pi \leq \frac{h_{i i}}{I_{i} g_{i 0}} U_{i}^{\prime}\left(\frac{P_{a_{i}} h_{i i}}{n_{0} B_{a_{i}} g_{i a_{i}}}\right) \\ \pi=\frac{h_{i i} U_{i}^{\prime}\left(\gamma_{i}\right)}{I_{i} g_{i 0}} & \text { if } \frac{h_{i i}}{I_{i} g_{i 0}} U_{i}^{\prime}\left(\frac{P_{a_{i}} h_{i i}}{n_{0} B_{a_{i}} g_{i a_{i}}}\right)<\pi<\frac{h_{i i}}{I_{i} g_{i 0}} U_{i}^{\prime}(0)(6) \\ b_{i}=0 & \text { if } \pi \geq \frac{h_{i i}}{I_{i} g_{i 0}} U_{i}^{\prime}(0)\end{cases}
$$

Noticing the structural similarity between (6) and (22) in [10], we can establish the existence of $\mathrm{NE}$ and the convergence to the NE under the best response update (6).

Definition 2. The PU selection game, denoted as $G_{N S A}^{P U}$ and $G_{N P A}^{P U}$ for the SINR and power auction respectively, is a tuple $\left(\mathcal{M}, \mathcal{A}=\left\{A_{i}\right\},\left\{\widehat{S}_{i}, i \in \mathcal{M}\right\}\right)$, where $\mathcal{M}$ is the player set,
$A_{i}=\mathcal{N}$ is the strategy set of $S U i$, the utility function of $S U i$ is defined as $\widehat{S}_{i}\left(a_{i}, a_{-i}\right) \triangleq S_{i}\left(\mathbf{a}, \mathbf{b}^{*}\right)$ where $\mathbf{b}^{*}(\mathbf{a})=$ $\left\{b_{i}^{*}(\mathbf{a}), i \in \mathcal{M}\right\}$ denotes the $N E$ of the bidding game under the PU setting a. Each player $(S U) i$ selects its strategy $(P U)$ $a_{i} \in \mathcal{N}$ to maximize its utility $\widehat{S}_{i}$.

To analyze the PU selection game, we write the optimization problem of each SU $i$ as

$$
\max _{a_{i}} \widehat{S}_{i}\left(a_{i}, a_{-i}\right)=\max _{a_{i}} S_{i}\left(\mathbf{a}, \mathbf{b}^{*}(\mathbf{a})\right) .
$$

Noticing that in the bidding game under PU setting a, it holds that $S_{i}\left(\mathbf{a}, \mathbf{b}^{*}(\mathbf{a})\right)=\max _{b_{i}} S_{i}\left(a_{i}, b_{i}\right)$, we thus have

$$
\max _{a_{i}} \widehat{S}_{i}\left(a_{i}, a_{-i}\right)=\max _{a_{i}} \max _{b_{i}} S_{i}\left(a_{i}, b_{i}\right),
$$

which, according to Lemma 1, is the same optimization problem as for the global auction game analyzed previously. Hence, we can map the NE of the PU selection game and the corresponding bidding game to the NE of the global auction game, as stated in the following theorem.

Theorem 3. Any (pure) NE of the auction game can be mapped to a (pure) NE of the PU selection game $\mathbf{a}^{*}$ and the corresponding $N E$ of the bid game $\mathbf{b}^{*}\left(\mathbf{a}^{*}\right)$ under the $P U$ setting $\mathrm{a}^{*}$, i.e., any pure NE of the power auction game can be expressed as $\mathbf{s}^{*}=\left(a_{i}^{*}, b_{i}^{*}\left(\mathbf{a}^{*}\right), i \in \mathcal{M}\right)$.

By decomposing the global auction game into the PU selection game and the bidding game, we introduce a two-level architecture into the spectrum auction problem, in which the higher level PU selection game is a finite strategy game. This hierarchicalization can help us analyze the spectrum auction in more complex scenarios, as explored in the next section.

\section{SPECTRUM AUCTION With FREE SPECTRUM BANDS}

Until now, we have analyzed the spectrum auction game in which the unlicensed SUs purchase spectrum ressource from licensed PUs. In this section, we extend our auction framework to the more challenging scenario with free spectrum bands. In such context, the SUs have the choice between accessing the licensed spectrum bands owned by PUs which is charged as a function of the enjoyed SINR or received power at PUs, and switching to the unlicensed spectrum bands which are free of charge but become more crowded when more SUs operate in these spectrum bands. Consequently, the SUs should strike a balance between accessing the free spectrum bands with probably more interference and paying for communication gains by staying with the licensed bands. In the subsequent study, we assume that there is one free spectrum band available for all SUs. The extension to multiple free band case is straightforward.

We start with the SINR auction. In the new scenario with a free band, we define the spectrum band set $\mathcal{N}=$ $\{1, \cdots, N, N+1\}$ where band 1 to $N$ are the licensed bands processed by PU 1 to $N$, band $N+1$ denotes the free band with bandwidth $B_{N+1}$. Compared with the previous analysis without free spectrum band, each SU $i$ has an additional choice of switching to band $N+1$ and the corresponding utility is

$$
S_{i}(N+1)=U_{i}\left(\gamma_{i}\right) \text {, }
$$

where $\gamma_{i}$ is the SINR of SU $i$. It is obvious to see that all SUs operating at $B_{N+1}$ transmits at its maximum power, denoted 
as $p_{j}^{\max }, j \in \mathcal{M}$, to maximize their utility. Hence

$$
\gamma_{i}=\frac{p_{i}^{\max } h_{i i}}{n_{0} B_{N+1}+\sum_{j \neq i, a_{j}=N+1} p_{j}^{\text {max }} h_{j i}} .
$$

From Corollary 1, each SU $i$ faces the choice of accessing the licensed band with minimum effective price and the free band $N+1$. As in Definition 1 and 2, we can define the corresponding PU selection game and bidding game in the new context ${ }^{6}$. The PU selection game is a finite strategy game and hence has at least one pure or mixed NE. By performing the same analysis as that in Section III-C, we can establish a mapping between a NE of the auction game and a NE of the PU selection game in the new context.

We then address the problem of how to reach a NE of the PU selection game, which is also a NE of the global auction game. We first notice that the myopic best response update in the PU selection game is not guaranteed to converge to a NE. In fact, during the course of PU selection, the SUs may notice that the utility of accessing a licensed spectrum is higher than staying in the free spectrum, and thus switch to the licensed spectrum accordingly. Since the SUs do this simultaneously, the free spectrum becomes under-loaded and the SUs will switch back to the free spectrum in the next iteration. This phenomenon, in which a player keeps switching between two strategies, is known as ping-pong effect.

To eliminate the ping-pong effect, we develop an algorithm based on the no-regret learning to converge to a correlated equilibrium (CE) of the PU selection game, which is shown to be a $\mathrm{CE}$ of the global auction game, too. Before presenting the proposed algorithm, we first provide a brief introduction on $\mathrm{CE}$ and no-regret learning.

\section{A. Overview of correlated equilibrium}

The concept of CE was proposed by Nobel Prize winner, Robert J. Aumann [5], in 1974. It is more general than NE. The idea is that a strategy profile is chosen randomly according to a certain distribution. Given the recommended strategy, it is to the players' best interests to conform with this strategy. The distribution is called CE, formally defined as follows.

Definition 3. Let $G=\left(\mathcal{N},\left(\Sigma_{i}, i \in \mathcal{N}\right),\left(S_{i}, i \in \mathcal{N}\right)\right)$ be a finite strategy game, where $\mathcal{N}$ is the player set, $\Sigma_{i}$ is the strategy set of player $i$ and $S_{i}$ is the utility function of $i, a$ probability distribution $p$ is a correlated equilibrium of $G$ if and only if $\forall i \in \mathcal{N}, r_{i} \in \Sigma_{i}$, it holds that

$$
\sum_{r_{-i} \in \Sigma_{-i}} p\left(r_{i}, r_{-i}\right)\left[S_{i}\left(r_{i}^{\prime}, r_{-i}\right)-S_{i}\left(r_{i}, r_{-i}\right)\right] \leq 0, \forall r_{i}^{\prime} \in \Sigma_{i},
$$

or equivalently,

$$
\sum_{r_{-i} \in \Sigma_{-i}} p\left(r_{-i} \mid r_{i}\right)\left[S_{i}\left(r_{i}^{\prime}, r_{-i}\right)-S_{i}\left(r_{i}, r_{-i}\right)\right] \leq 0, \forall r_{i}^{\prime} \in \Sigma_{i} .
$$

The second formula means that when the recommendation to player $i$ is to choose action $r_{i}$, then choosing action $r_{i}^{\prime} \neq r_{i}$ cannot lead to a higher expected payoff to $i$.

The CE set is nonempty, closed and convex in every finite strategy game. Moreover, every NE is a CE and corresponds

\footnotetext{
${ }^{6}$ For the free band, there is no bidding game, or alternertively, we can define a dumb bidding game for the free band, at the NE of which each SU choosing the free band submits 0 as bid and the utility is given by (7)
}

to the special case where $p\left(r_{i}, r_{-i}\right)$ is a product of each individual player's probability for different actions, i.e., the play of the different players is independent.

\section{B. Overview of no-regret learning}

The no-regret learning algorithm [4] is also termed regretmatching algorithm. The stationary solution of the no-regret learning algorithm exhibits no regret and the probability of choosing a strategy is proportional to the "regret" for not having chosen other strategies. For any two strategies $r_{i} \neq r_{i}^{\prime}$ at any time $T$, the regret of player $i$ for not playing $r_{i}^{\prime}$ is

$$
R_{i}^{T}\left(r_{i}, r_{i}^{\prime}\right) \triangleq \max \left(D_{i}^{T}\left(r_{i}, r_{i}^{\prime}\right), 0\right),
$$

where

$$
D_{i}^{T}\left(r_{i}, r_{i}^{\prime}\right) \triangleq \frac{1}{T} \sum_{t \leq T}\left(S_{i}^{t}\left(r_{i}^{\prime}, r_{-i}\right)-S_{i}^{t}\left(r_{i}, r_{-i}\right)\right)
$$

$D_{i}^{T}\left(r_{i}, r_{i}^{\prime}\right)$ has the interpretation of average payoff that player $i$ would have obtained, if it had played $r_{i}^{\prime}$ every time in the past instead of $r_{i} . R_{i}^{T}\left(r_{i}, r_{i}^{\prime}\right)$ is thus a measure of the average regret. The probability that player $i$ chooses $r_{i}$ is a linear function of the regret. For every period $T$, define the relative frequency of players' strategy $\mathbf{r}$ played till $T$ periods of time as follow:

$$
z_{T}(\mathbf{r}) \triangleq \frac{1}{T} N(T, \mathbf{r})
$$

where $N(T, \mathbf{r})$ denotes the number of periods before $T$ that the players' strategy is r. As an important property, $z_{T}$ is guaranteed to converge almost surely (with probability one) to a set of $C E$ in no-regret learning algorithm.

\section{Proposed algorithm based on no-regret learning}

In this subsection, we develop an algorithm (Algorithm 2) based on no-regret learning and prove its convergence to a $\mathrm{CE}$ of the SINR auction game.

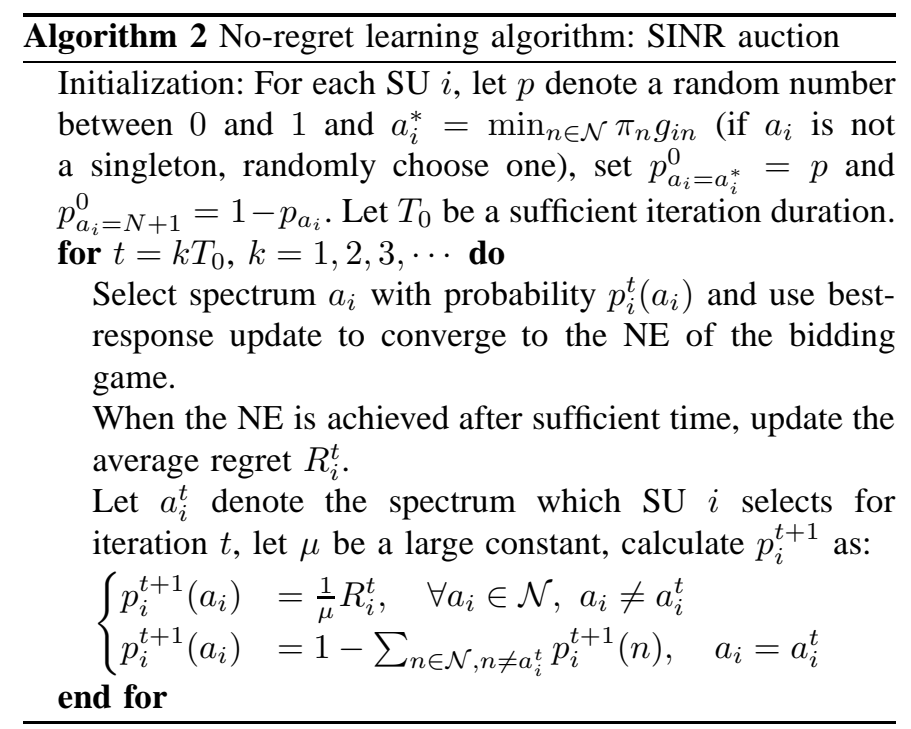

Theorem 4. There exists a threshold price vector $\pi^{\text {th }}$ such that if the price vector $\pi>\pi^{t h}$, the proposed algorithm converges surely to a CE of the SINR auction game.

Proof: It follows from the structure of the bidding game that a threshold price vector $\pi^{t h}$ exists such that if the 
price vector $\pi>\pi^{t h}$, the convergence to the NE of the bidding game is guaranteed under the given spectrum setting. It then follows from the convergence property of the no-regret learning that the proposed algorithm converges surely to a $\mathrm{CE}$ of the PU selection game, denoted as p, i.e.,

$$
\begin{gathered}
\sum_{a_{j} \in \mathcal{N}, j \in \mathcal{M}, j \neq i} p\left(a_{-i} \mid a_{i}\right)\left[S_{i}\left(\left(a_{i}^{\prime}, b_{i}^{\prime *}\right),\left(a_{-i}, b_{-i}^{\prime}{ }^{*}\right)\right)-\right. \\
\left.S_{i}\left(\left(a_{i}, b_{i}^{*}\right),\left(a_{-i}, b_{-i}^{*}\right)\right)\right] \leq 0, \quad \forall a_{i}^{\prime} \in \mathcal{N},
\end{gathered}
$$

where $b_{i}^{*}$ and $b_{i}^{\prime *}$ is the strategy of SU $i$ at the NE of the bidding game under the spectrum setting $\left(a_{i}, a_{-i}\right)$ and $\left(a_{i}^{\prime}, a_{-i}\right)$, respectively. It follows from the $\mathrm{NE}$ definition of the bidding game that

$$
S_{i}\left(\left(a_{i}^{\prime}, b_{i}^{\prime *}\right),\left(a_{-i}, b_{-i}^{\prime}{ }^{*}\right)\right)=\max _{\gamma_{i}} U_{i}\left(\gamma_{i}\right)-\pi_{a_{i}^{\prime}} g_{i a_{i}^{\prime}} \gamma_{i} .
$$

On the other hand, we have

$$
S_{i}\left(\left(a_{i}^{\prime}, b_{i}^{\prime}\right),\left(a_{-i}, b_{-i}^{*}\right)\right) \leq \max _{\gamma_{i}} U_{i}\left(\gamma_{i}\right)-\pi_{a_{i}^{\prime}} g_{i a_{i}^{\prime}} \gamma_{i}, \forall b_{i}^{\prime} \geq 0 .
$$

Hence, it holds that

$$
\begin{aligned}
& \sum_{a_{j} \in \mathcal{N}, j \in \mathcal{M}, j \neq i} p\left(a_{-i} \mid a_{i}\right)\left[S_{i}\left(\left(a_{i}^{\prime}, b_{i}^{\prime}\right),\left(a_{-i}, b_{-i}^{*}\right)\right)-\right. \\
& \left.\quad S_{i}\left(\left(a_{i}, b_{i}^{*}\right),\left(a_{-i}, b_{-i}^{*}\right)\right)\right] \leq 0, \quad \forall a_{i}^{\prime} \in \mathcal{N}, \forall b_{i} \geq 0,
\end{aligned}
$$

indicating that $\mathbf{p}$ is also a CE of the SINR auction game.

As a desirable property, Algorithm 2 can be implemented distributedly such that each SU $i$ only needs to know the price vector $\pi$, its own channel gain $h_{i i}$ and that between $S_{i}$ and each PU $n g_{i n}$. The best response update of the bidding game can be implemented distributedly at each SU $i$ based on the knowledge of $h_{i i}$ and $g_{i n}$, the measurement of $n_{0}$ and the SINR $\gamma_{i}$, as detailed in [10]. We then show that the average regret can be calculated at each SU without any other information. Noticing (9) and recall the utility function of the PU selection game in Definition 2, it suffices to show that at each iteration $t, \Gamma_{i}^{t}\left(a_{i}^{t}, a_{-i}^{t}\right) \triangleq \sum_{k \leq t} \widehat{S}_{i}\left(a_{i}^{t}, a_{-i}^{k}\right), \forall a_{i}^{t} \in \mathcal{N}$ can be calculated distributedly.

In fact, at each iteration $k, \widehat{S}_{i}$ can be calculated as

$$
S_{i}^{k}=\left\{\begin{array}{ll}
U_{i}\left(\frac{h_{i i} p_{i}^{\max }}{I_{i}^{N+1}}\right) & a_{i}^{t}=N+1 \\
U_{i}\left(\gamma_{i a_{i}^{t}}^{*}\right)-\pi_{a_{i}^{t}} g_{i a_{i}^{t}} \gamma_{i a_{i}^{t}}^{*} & a_{i}^{t} \neq N+1
\end{array},\right.
$$

where $\gamma_{i a_{i}^{t}}^{*}=U_{i}^{\prime-1}\left(\pi_{a_{i}^{t}} g_{i a_{i}^{t}}\right), I_{i}^{N+1}$ is the interference experienced by SU $i$ when choosing the free band, which can be measured locally. $\Gamma_{i}^{t}$ can then be calculated by induction as

$$
\Gamma_{i}^{t}=\left\{\begin{array}{ll}
U_{i}^{t}\left(a_{i}^{t}, a_{-i}^{t}\right) & t=1 \\
\Gamma_{i}^{t-1}\left(a_{i}^{t}, a_{-i}^{t-1}\right)+U_{i}^{t}\left(a_{i}^{t}, a_{-i}^{t}\right) & t>1
\end{array} .\right.
$$

Consequently, the average regret can then be calculated based on only local measurement, which leads to the entirely distributed implementation of the proposed algorithm.

For the power auction, a similar distributed algorithm based on no-regret learning can be derived with convergence to a CE.

\section{Simulation Analysis}

In this section, we conduct simulations to evaluate the performance of the proposed auction framework and demonstrate some intrincical properties of the proposed auction framework, especially the fairness and efficiency, which are not explicitely addressed in the analytical part of the paper. After presenting the simulation setting, we introduce a reference power allocation scheme, called NAIVE, to which our proposed auction

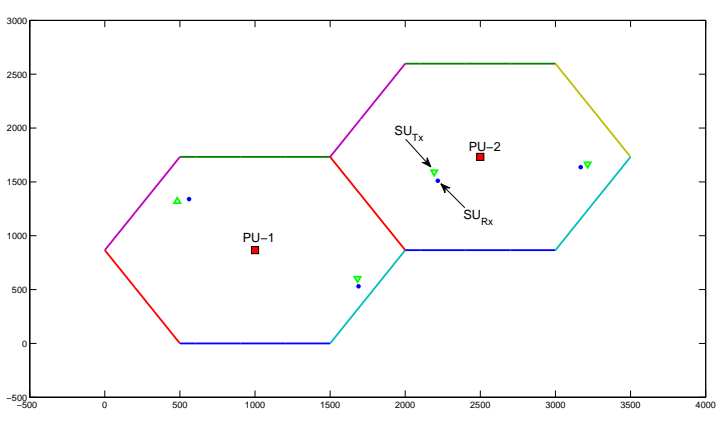

Fig. 1. Simulation setting

mechanisms are compared. In the first set of simulations, we consider an illustrative scenario to compare the SINR, power auctions with the NAIVE scheme. In the second set of simulations, we focus on the power auction in realistic network configurations with and without free spectrum band.

\section{A. Simulation parameters and reference scheme}

In our simulations, we consider a network of two PUs and multiple SUs (transmitter-receiver pairs). PUs can be seen as two access points or base stations covering two hexagonal cells, as shown in Figure 1. They can accept a certain amount of interference while allowing SUs to communicate during uplink PU transmissions.

In all simulations, we set $B_{n}=5 \mathrm{MHz}$ and $P_{n}=2 n_{0} B_{n}$ $\forall n$. We adopt a typical urban path-loss model (C2 NLOS WINNER model [15] for WiMAX) with carrier frequency $f_{c}=3.5 \mathrm{GHz}$ and path-loss exponent $\alpha=3.5$. Shadowing effect is neglected.

In order to show the performance gain brought by our solutions, we introduce a reference power allocation scheme termed NAIVE. In NAIVE, SUs choose the furthest PU based on the knowledge of channel gains $g_{i n}{ }^{7}$. Each PU $n$ then allocates power $p_{i}=P_{n} /\left(M_{n} g_{i n}\right)$ to $\mathrm{SU} i$ choosing it, where $M_{n}$ is the number of SU choosing PU $n$. In the scenario with a free band, the SUs in the NAIVE scheme switch to the free band with certain probability $p_{\text {free }}$ (we analyze the cases $p_{\text {free }}=1 / 2$ and $p_{\text {free }}=1 / 3$ ). This simple scheme serves as the reference scheme for performance comparison.

\section{B. Illustrative example: SINR and power auctions}

We start with an illustrative example to compare the SINR, power auctions and the NAIVE scheme. We consider the fixed network configuration illustrated in Figure 1 with two PUs and four SUs with $\theta_{i} \in[1,20], \forall i$. There is no free band in this example. The prices $\pi_{1}=\pi_{2}$ are optimized by dichotomy ${ }^{8}$.

We study the dynamics of the spectrum acution game under the best-response update. In the SINR auction, each SU chooses the PU with the minimum effective price (cf. Corollary 1) and then iteratively adjust its bid. Figure 2 (left) shows the convergence of allocated power to SUs. After about 40 iterations, convergence is reached. Compared with the

\footnotetext{
${ }^{7}$ The rationale of the choice is that choosing the furthest PU causes the least interference at the PU.

${ }^{8}$ Recall that the more competitive scenario where the PUs set their prices to maximize their revenue consists of a significant extension of the current work and is left for future studies.
} 

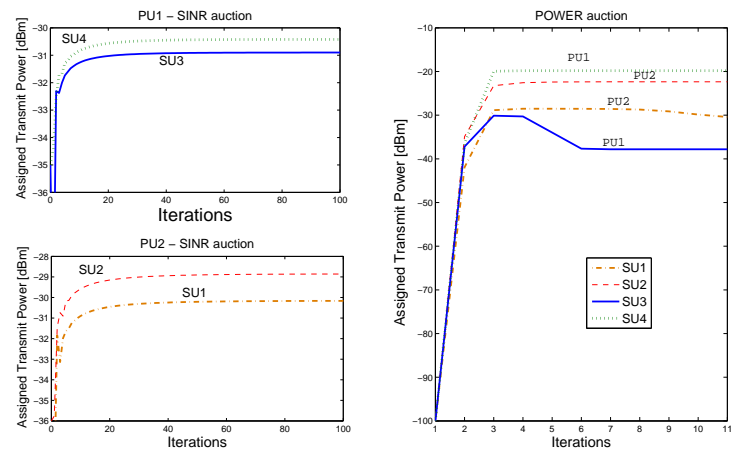

Fig. 2. Convergence of allocated power in the SINR auction (left) and power auction (right); final PU choice is shown for each SU with power auction.
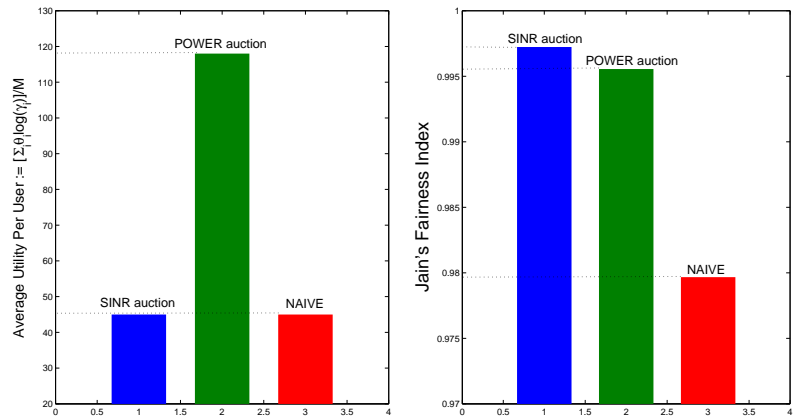

Fig. 3. Average utility per SU (left) and Jain's fairness index calculated based on $U_{i} / \theta_{i}$ (right) for SINR, power auctions and NAIVE

SINR auction where the choice of PU is done at the very first iteration and is not modified afterwards, in the power auction, the effective price is given by Lemma 3. As one part of the effective price, $I_{i}$ changes from one iteration to another depending on the strategy of other SUs $s_{-i}$, thus the choice of PU may also vary from one iteration to another. However, as shown in Figure 2 (right), the final allocated power of each SU converges after about 10 iterations and the choice of PU is stablized. Compared with the SINR auction, the power auction converges in a faster but less smooth way.

In Figure 3, we focus on the efficiency and fairness of the considered schemes by studying the average utility per SU and the Jain's fairness index [16]. The Jain's index is computed based on the normalized utility $U_{i} / \theta_{i}$. From the results, we observe that the SINR auction and the NAIVE scheme have almost the same average utility, but the SINR auction outperforms significantly the NAIVE scheme in terms of fairness. The power auction, on the other hand, has a very good performance in terms of both efficiency and fairness.

\section{Realistic experiment: power auction}

We now turn to more realistic scenarios. We focus on the power auction as it achieves the best performance in the above illustrative exemple. The power auction is also more natural and realistic in that SUs pay for the interference they create to PUs instead of the SINR they get as in the SINR auction. In our simulation, the transmitters of SUs are randomly located in each of the two cells. the receivers are randomly drawn in a disk with radius $100 \mathrm{~m}$ whose center is the corresponding transmitter. We run Monte Carlo simulations with 1000 snapshots. At each snapshot, SU locations are randomly drawn with $\theta_{i}$ randomly drawn in $[1,20]$.

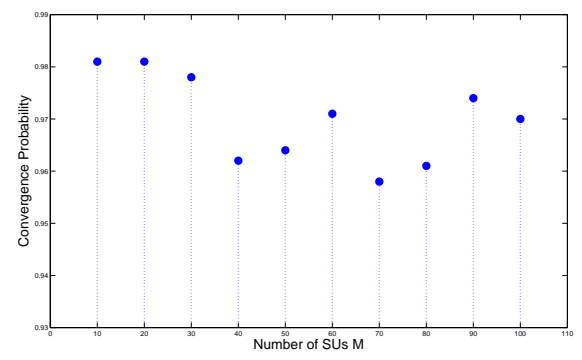

Fig. 4. Convergence probability of power auction

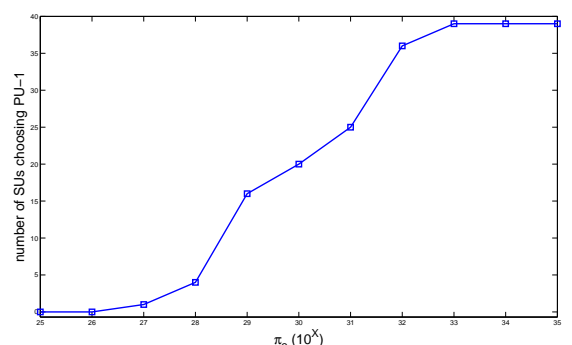

Fig. 5. Number of SUs choosing PU 1 as a function of $\pi_{2}$

1) Convergence: As explained in Section III-B, the bestresponse update is not guaranteed to converge. We thus study the convergence probability. We consider that the convergence is achieved if the best-response update in the power auction converges within 100 iterations, otherwise we consider that the auction does not converge. Figure 4 shows the probability of convergence as a function of the number of SU under this criterion: in the vast majority cases (more precisely, in more than $95 \%$ cases), convergence is achieved. In the subsequent simulations, in case of non-convergence, the results are based on the allocated power values after 100 iterations.

2) Load balancing: Figure 5 shows a scenario in which PU 1 fixes its price $\pi_{1}=10^{30}$ and PU 2 varies its price $\pi_{2}$ in the range $\left[10^{25}, 10^{35}\right]$. The total number of SUs $M$ is set to 40. As shown in the figure, the number of SUs choosing PU 1 increases with $\pi_{2}$. The results demonstrate the benefit of the proposed power auction framework in load balancing by adjusting the prices of PUs. This feature is obviously not possible in NAIVE.

3) Efficiency and fairness: We now focus on two key performance metrics: efficiency and fairness. To this end, we compare the power auction and the NAIVE scheme in terms of average utility per SU and the Jain fairness index in two configurations. In the first configuration $M / 2$ system, half of SUs are geographically located in cell 1 and the other half in cell 2 . In the second configuration $M-2$ system, the number of SUs in cell 2 is constant $\left(M_{2}=2\right)$, while the number of SU in cell 1 is variable in cell $1\left(M_{1}=M-2\right)$. The two configurations represent two typical network scenarios, the balanced one with a homogeneous distribution of SUs and the unbalanced one with a heterogeneous distribution of SUs. As for the illustrative example, we set $\pi_{1}=\pi_{2}$ and choose the price by dichotomy for the given number of SUs.

Figure 6 (left) shows that the average utility per SU is almost the same in the two configurations in the power auction (see the $\mathrm{M} / 2=\mathrm{M}-2$ MultiPU Power curve in the figure) and is always higher than that in the NAIVE scheme. Figure 7 shows that the Jain fairness index (calculated in the same way as in the illustrative example) of power auction is always above that 

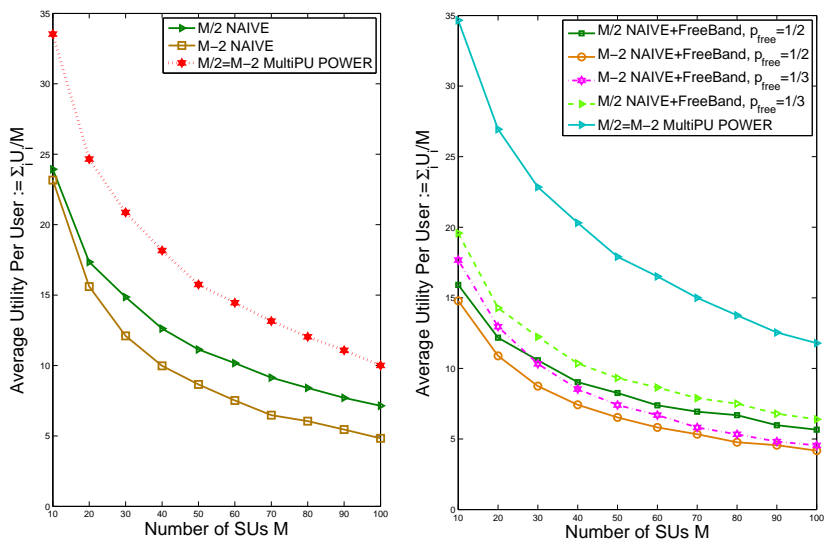

Fig. 6. Utility comparison in balanced $(M / 2)$ and unbalanced $(M-2)$ scenarios between power auction and NAIVE with (left) and without (right) free band

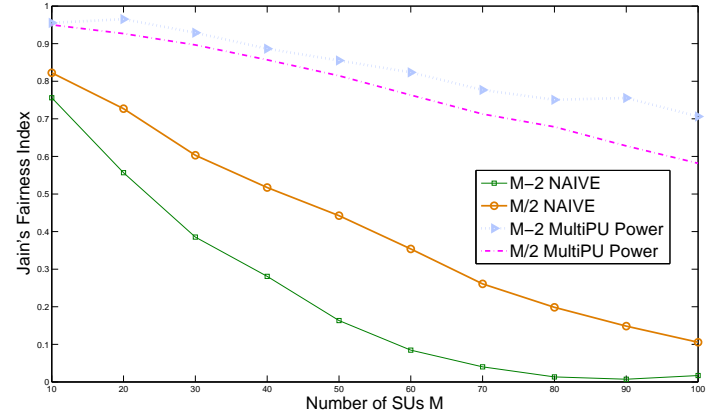

Fig. 7. Fairness comparison in balanced $(M / 2)$ and unbalanced $(M-2)$ scenario between power auction and NAIVE

of NAIVE. In particular, in the unbalanced scenario, the power auction outperforms significantly the NAIVE scheme.

4) Power auction with a free band: We now study the power auction and the proposed no-regret learning algorithm (Section IV-C) by introducing a free band of $5 \mathrm{MHz} . p_{i}^{\max }=$ $20 \mathrm{dBm}, \forall i \in \mathcal{M}$. In the simulation, SUs in the NAIVE scheme choose the free band with probability $p_{\text {free }}=1 / 2$ or $p_{\text {free }}=1 / 3$ and emit at the maximum power $p_{i}^{\max }$. The power allocation of SUs staying in licensed bands follows the same way as in the scenario without free band.

Figure 6 (right) shows the average utility of the power auction and NAIVE. As can be observed, compared with the scenario without free band, the average utility in NAIVE is slightly degraded even a new band is introduced. In contrast, the no-regret learning algorithm results a higher utility when the free band is added. Consequently, the utility gap between the power auction and NAIVE is more significant in the scenario with free band. Furthermore, we observe the convergence of the no-regret learning algorithm. Figure 8 shows the evolution of the number of SUs choosing PU1, PU2 and the free band for $M=50$. The results demonstrate the benefit of the proposed no-regret learning algorithm to converge to an equilibrium with reasonable network efficiency in a distributed fashion.

\section{CONCLUSION}

In this paper, we proposed an auction framework for cognitive radio networks to allow unlicensed SUs to share the available spectrum of licensed PUs, subject to the interference temperature constraint at each PU. We provided an in-

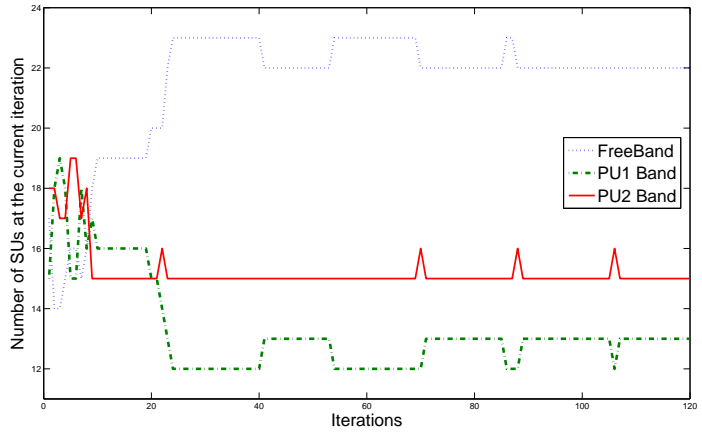

Fig. 8. Evolution of number of SUs choosing PU1, PU2 and the free band

depth analysis on the resulting multiple-PU multiple-SU noncooperative auction game. We then extended the proposed auction framework to the more challenging scenario with free spectrum bands by developing an algorithm based on no-regret learning to reach a $\mathrm{CE}$ of the auction game. The proposed algorithm, which can be implemented distributedly based on local observation, is especially suited in decentralized adaptive learning environments as cognitive radio networks. The simulation results demonstrate the effectiveness of the proposed auction framework in achieving high efficiency and fairness in spectrum allocation.

As stated in the paper, a significant extension of our work is to study the more competitive Stackelberg game in which PUs choose their prices to maximize their revenue. Studying the efficiency of the spectrum auction in that scenario is the subjet of our on-going work.

\section{REFERENCES}

[1] S. Haykin. Cognitive radio: brain-empowered wireless communications. IEEE Journal on Selected Areas in Communications, 23(2):201-220, 2005.

[2] M. Buddhikot. Understanding dynamic spectrum allocation. In Proc. IEEE DySPAN, April 2007.

[3] Spectrum policy task force report. Federal Communications Commission, Nov., 2002.

[4] S. Hart and A. Mas-Colell. A simple adaptive procedure leading to correlated equilibrium. Econometrica, 68(5):1127-1150, 2000.

[5] R. J. Aumann. Subjectivity and correlation in randomized strategy. Journal of Mathematical Economics, 1(1):67-96, 197.

[6] V. Krishna. Auction Theory. Academic Press, 2002.

[7] X. Zhou and H. Zheng. Trust: A general framework for truthful double spectrum auctions. In Proc. IEEE Infocom, 2009.

[8] J. Zhu and K. J. R. Liu. Multi-stage pricing game for collusionresistant dynamic spectrum allocation. IEEE Journal on Selected Areas in Communications, 26(1):182-191, Jan 2008.

[9] Gaurav S. Kasbekar and S. Sarkar. Spectrum auction framework for access allocation in cognitive radio networks. In Proc. ACM Mobihoc, 2009.

[10] J. Huang, R. Berry, and M. L. Honig. Auction-based spectrum sharing. Mobile Networks and Applications (MONET), 11:405-418, 2006.

[11] S. Gandhi, C. Buragohain, L. Cao, H. Zheng, and S. Suri. A general framework for wireless spectrum auctions. In Proc. IEEE DySPAN, April 2007.

[12] Y. Wu, B. Wang, K. J. R. Liu, and T. Charles Clancy. A scalable collusion-resistant multi-winner cognitive spectrum auction game. IEEE Transactions on Communications, to appear.

[13] R.B. Myerson. Game Theory: Analysis of Conflict. Harvard University Press, Cambridge, MA, 1991.

[14] J. B. Rosen. Existence and uniqueness of equilibrium points for concave n-person games. Econometrica, 33(3):520-534, Jul 1965.

[15] Roshni Srinivasan. IEEE 802.16m Evaluation Methodology Document (EMD). IEEE, 2008.

[16] R. Jain, D. Chiu, and W. Hawe. A quantitative measure of fairness and discrimination for resource allocation in shared computer systems. DEC Research Report TR-301, 1984. 